\title{
Small is even more different now
}

\author{
We celebrate the fifteenth anniversary of Nature Nanotechnology by looking at how the journal topics have \\ expanded throughout the years.
}

T he fiery red cover of the first issue of Nature Nanotechnology introduced a superconducting quantum interference device made with carbon nanotubes to the community of researchers in nanoscience and nanotechnology: "welcome to the nanoSQUID". Carbon nanotubes were indeed the great protagonist of research at the nanoscale back in 2006, and five of the seven articles included in the first issue were based on these materials.

Fast-forward fifteen years and the variety of nanomaterials covered by articles in the journal is considerably more diverse. We have often attempted to list them all in some sort of directory to appear in our journal scope, always with scarce success. We have eventually realized that our journal is simply about every specimen of material small enough to have properties different from those of its bulk correspondent due to one or more of its dimensions being at the nanoscale. The research in nanomaterials can broadly be divided into four main themes, namely synthesis and characterization, fundamental properties, applications, and implications for the environment and health.

To mark the 15th anniversary each of the editors of Nature Nanotechnology, plus a colleague from Nature Communications, has selected one research paper that in their view marked an important milestone in a specific field. As often happens in these cases, every choice is too limiting. However, it is interesting to see how a selection purely dictated by personal taste illustrates so well the four themes mentioned above. Olga Bubnova highlighted the achievement of large-scale synthesis of graphene aimed at industrial applications. Benjamin Heinrich chose two reports on the generation of magnetic skyrmions that fall in the category of fundamental properties of nanomaterials. In the article selected by Chiara Pastore, both fundamental and applicative aspects of nanoparticles used as therapeutic agents are important. Similarly, Gabriella Graziano recounted the proposal, based on fundamental insights, of a device based on metal nanostructures and aimed at applications in water splitting. Wenjie Sun and Silvia Milana picked papers on applications in gas separation and digital electronics, respectively. Finally, Fabio Pulizzi highlighted the results reported in an analysis of nanomaterials intended for applications in agriculture that focused both on efficacy and implications for the environment.

As we did on the occasion of the 10th anniversary of the journal, we would like to thank all editors that have contributed with their enthusiasm and hard work in making the journal so successful and have now left. In addition to those already mentioned we are grateful to Ai Lin Chun, Owain Vaughan, Bryden Le Bailly, Giacomo Prando, Christine Horejs and Gabriella Graziano. As always we are only too aware that the success of the journal is primarily due to all of you: authors, reviewers and readers. As editors, we shall continue to do our best to publish the most significant results in the field and we shall make efforts to improve the service that we provide. In the meantime, to celebrate with you we have asked some experts in various fields of nanoscience and nanotechnology to join us in a series of webinars and tell us all about the state of the art of their fields. We invite you to attend and hope you will enjoy these free events.

Published online: 8 October 2021 https://doi.org/10.1038/s41565-021-01013-Z

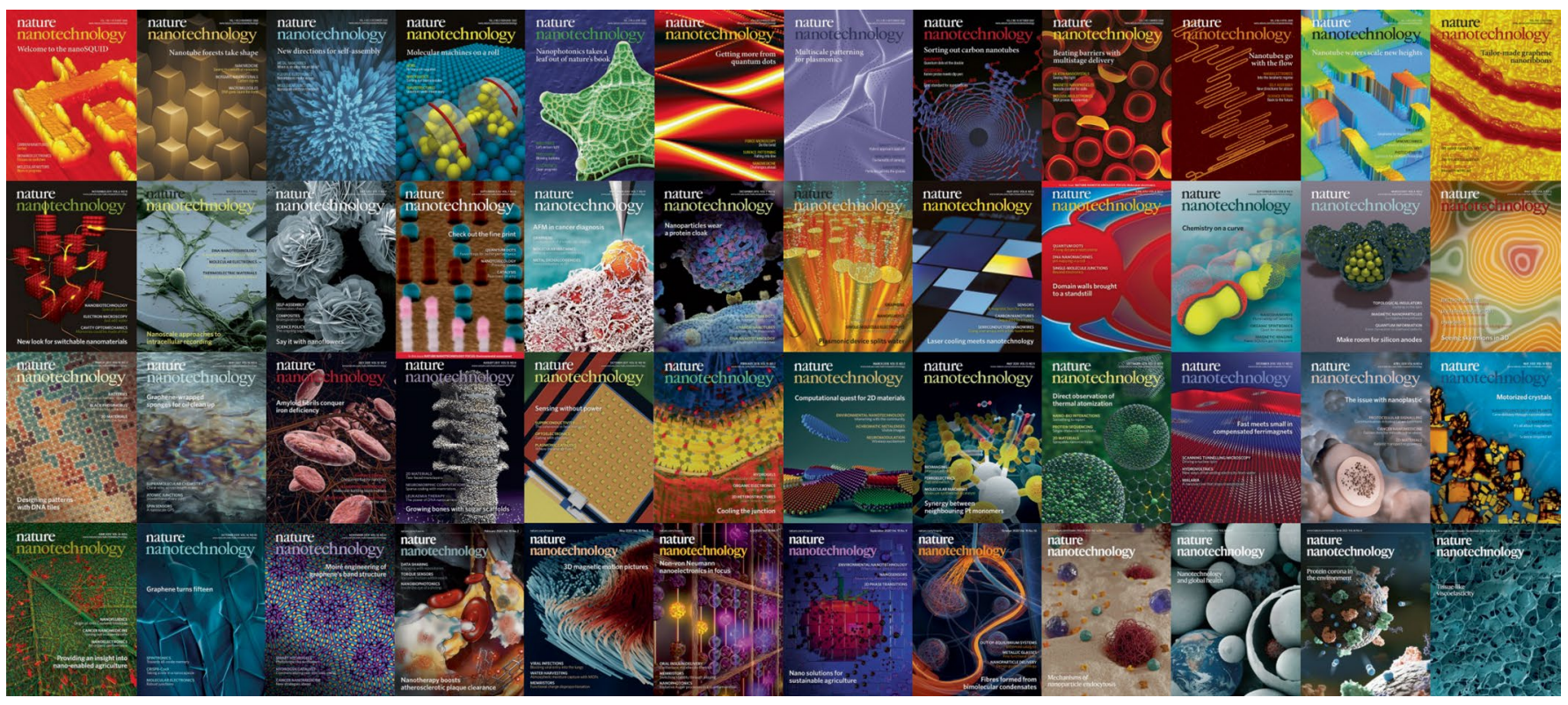

